\title{
Evaluación del dolor crónico en una población de pacientes hemodializado
}

XVII Premio de Investigación en Enfermería Nefrológica Janssen-Cilag

\author{
Miguel Ángel Rodríguez Calero - Daniel Hernández Sánchez - Ma José Gutiérrez Navarro \\ Francisca Juan Amer - Jordi Calls Ginesta
}

Unidad de Hemodiálisis. Fundación Hospital Manacor, Mallorca

\section{Resumen}

El dolor es un síntoma frecuente en los pacientes sometidos a hemodiálisis, influyendo negativamente en su calidad de vida. La mayor accesibilidad a la terapia dialítica, el mayor tiempo de permanencia de los pacientes en los programas de hemodiálisis y el envejecimiento de la población han contribuido a la presencia habitual del dolor en la vida de estas personas. En el presente estudio se evalúa el dolor crónico en una población de hemodiálisis usando instrumentos de valoración validados procedentes de otras disciplinas, que han sido recientemente utilizados en pacientes de hemodiálisis.

Un $82.1 \%$ de nuestros pacientes afirmaron tener dolor en casa, únicamente 7 pacientes lo negaron, y sólo en el $28.12 \%$ de los casos, la Escala Visual Analógica (EVA) usada para averiguar su intensidad, fue 0 .

\section{Correspondencia: \\ Miguel Ángel Rodríguez Calero \\ Unidad de Hemodiálisis \\ Fundación Hospital Manacor \\ Ctra. Manacor-Alcudia s/n \\ 07500, Manacor, Islas Baleares \\ mianrodriguez@hospitalmanacor.org}

Medidas no farmacológicas como el calor, masajes o cambios posturales mostraron una eficacia similar a la del tratamiento con fármacos.

El tiempo de permanencia en hemodiálisis y el valor de PTHi se relacionaron estadísticamente con la intensidad del dolor y la descripción cualitativa del mismo, alterando frecuentemente las actividades de la vida diaria de los pacientes, principalmente el desplazamiento/caminar y la actividad general. Estas relaciones, descritas por primera vez en la literatura científica, podrían explicar, en parte, la alta prevalencia de este síntoma y el alto porcentaje de dolor músculo-esquelético referido por nuestros pacientes.

\section{Palabras clave}

DOLOR

HEMODIÁLISIS

ESCALA VISUAL ANALÓGICA

ANALGESIA

\section{Evaluation of chronic pain in a population of} patients on haemodialysis

\section{Abstract}

Pain is a frequent symptom in patients undergoing dialysis, having a negative effect on their quality of 
life. Greater access to dialysis therapy, more time spent by patients on haemodialysis programmes and aging population have contributed to the habitual presence of pain in the life of these people. This study evaluates chronic pain in a population of patients on haemodialysis using validated valuation instruments used in other disciplines, which have been used recently in haemodialysis patients.

$82.1 \%$ of our patients indicated that they felt pain at home, only 7 patients denied having pain and only in $28.12 \%$ of cases the Visual Analogue Scale (VAS) used to determine the intensity of pain was zero.

Non-pharmacological measures such as heat, massages or changes in posture showed similar efficiency to treatment with drugs.

The time spent on haemodialysis and the value of PTHi were related statistically to the intensity of the pain and the qualitative description of it, frequently altering the activities of patients' daily lives, mainly moving/walking and general activity. These connections, described for the first time in scientific literature, could explain in part the high prevalence of this symptom and the high percentage of muscular-skeletal pain referred by our patients.

\section{Key words \\ PAIN \\ HAEMODIALYSIS \\ VISUAL ANALOGUE SCALE \\ ANALGESIA}

\section{Introducción}

A pesar del gran desarrollo científico de las disciplinas sanitarias y de la alta tecnificación de la medicina, el dolor sigue generando una gran preocupación tanto a los usuarios del sistema sanitario como a los profesionales, suponiendo un elevado gasto sanitario y social. Podemos definirlo como una experiencia emocional desagradable, una actividad perceptiva en la cual se integra la aferencia sensorial junto con va- riables psicológicas, sociales y culturales, y que está influenciada por factores individuales como las creencias, las estrategias de afrontamiento, la alteración emocional y otras. El dolor se presenta frecuentemente en la literatura científica como un complejo fenómeno de carácter universal, multicultural y multidimensional.

La aparición del dolor en la literatura científica en el campo de la hemodiálisis (HD) se enmarca en el estudio de la calidad de vida de los pacientes tratados con esta terapia, donde se ha asumido su protagonismo como síntoma altamente influyente en el bienestar de la persona.

La coexistencia de múltiples patologías en los pacientes con insuficiencia renal crónica terminal (IRCT), trae consigo síntomas de muy diversas naturalezas que se unen a otros provocados a medio y largo plazo por la propia HD, tales como patologías cardio-vasculares, isquémicas, neuropatías o enfermedades músculo-esqueléticas como la osteodistrofia renal, que suponen diferentes focos potenciales de dolor.

Asimismo, la mayor accesibilidad de la terapia hemodialítica, el envejecimiento de la población de HD y el mayor tiempo de permanencia en $\mathrm{HD}^{1}$ han contribuido a la presencia habitual de dolor en estos pacientes, en especial dolor crónico.

A pesar de su relevancia, el dolor ha sido durante años un gran olvidado dentro de la comunidad científica en el campo de la HD, siendo muy escasos los estudios publicados sobre este tema en nuestro ámbito. Sin embargo, existe un denominador común en ellos: es un síntoma altamente prevalente en estos pacientes, entre un $50 \%$ y un $98 \%$ de ellos lo padecen, y su manejo resulta en muchos casos insuficiente 0 inadecuado $^{2,3}$.

La valoración del dolor en el paciente de HD ofrece aún bastantes dudas debido a que no existen instrumentos diseñados específicamente para detectar las necesidades de estos pacientes. En estudios realizados en los últimos años ${ }^{2,3}$ se han utilizado escalas y cuestionarios validados para otras disciplinas, fundamentalmente para los cuidados paliativos del paciente oncológico. Hay que destacar la necesidad de 
profundizar en las necesidades valorativas del paciente en HD y en la descripción del dolor en este tipo de pacientes para sentar las bases de posteriores investigaciones dedicadas a su tratamiento individualizado y al diseño de planes de cuidados estandarizados para el manejo del dolor en pacientes en tratamiento renal sustitutivo.

El objetivo del presente estudio es describir el dolor crónico que padece la población en HD de nuestro centro, profundizando en sus características definitorias como temporalidad, causalidad, localización, severidad e influencia del tratamiento con hemodiálisis en el dolor, así como el papel de este síntoma en el desarrollo de la vida cotidiana de nuestros pacientes. También nos proponemos analizar la adecuación del tratamiento farmacológico que los pacientes reciben por parte del médico para mitigar el dolor, así como la adherencia a este tratamiento por parte del paciente. Para ello, se han usado escalas de medición utilizadas en recientes estudios, validadas y ampliamente usadas en la valoración del tratamiento y control del dolor crónico.

\section{Metodología}

Se trata de un estudio descriptivo y transversal, en el que se incluyeron todos los pacientes sometidos a HD durante más de un mes en nuestra unidad (38). Fueron excluidos aquellos pacientes que decidieron voluntariamente no tomar parte en el estudio (4) y aquellos que, por su estado mental o intelectual, no estaban en condiciones de responder correctamente a los cuestionarios que se les iban a presentar(2).

A los restantes 32 pacientes se les aplicaron tres herramientas de valoración del dolor, para lo cual fueron abordados fuera del ambiente de la sala de HD. El proceso de recogida de datos se llevó a cabo entre los meses de febrero y marzo de 2006.

El primer instrumento utilizado fue el Brief Pain Inventory (BPI) ${ }^{4}$, herramienta de referencia para la evaluación del dolor crónico, en el que se exploraron las principales dimensiones del dolor como su intensidad, interacción con las actividades de la vida diaria (AVDs), distrés psicológico o impacto en el aislamiento social.
En segundo lugar se aplicó el McGill Pain Questionnaire $(M P Q)^{5,6}$ en el que se evaluaron aspectos cuantitativos y cualitativos del dolor tales como su localización, intensidad, propiedades temporales y cualidad. Se trata de un cuestionario validado, ampliamente utilizado en diferentes grupos de pacientes con dolor crónico. De este cuestionario se obtuvieron a su vez tres índices:

- Número de palabras elegidas (NPE)

- Índice de valoración del dolor (PRI)

- Escala cualitativa de intensidad del dolor (PPI), en la que el paciente debe calificar el dolor en una escala entre "leve" (1 punto) a "insoportable" (5 puntos).

La intensidad del dolor presente fue evaluada a través de la Escala Visual Analógica (EVA), incluida en este cuestionario.

Debido a su complejidad, el MPQ fue administrado por un entrevistador.

Por último, se aplicó una encuesta complementaria especialmente diseñada para este estudio, en la que se valoraron aspectos del dolor directamente relacionados con la sesión de hemodiálisis y que no se encontraban en los anteriores instrumentos.

Otro marcador evaluado fue el Índice de Manejo del Dolor (Pain Management Index, PMI) 2,3,4, que relaciona la intensidad del dolor descrita por el paciente a través de la EVA con el nivel de analgesia prescrito, adquiriendo valores entre -3 y 3 . Puntuaciones negativas en el PMI indicaban tratamiento inadecuado y puntuaciones de 0 y superiores se consideraron una aproximación a un tratamiento aceptable. Este índice ha sido ampliamente utilizado en el control del dolor oncológico.

Se diseñó también la etiqueta de paciente "sufridor", teniendo en cuenta las recomendaciones de la Sociedad Española de Cuidados Paliativos (SEC$P A L)$, para definir aquellos pacientes con puntuaciones iguales o superiores a 3 en la EVA o en cualquier actividad de la vida diaria referida en el BPI.

El análisis estadístico se realizó utilizando el programa Graphpad Prism versión 2 (1995). Los resultados se expresan como media \pm desviación estándar. 


\section{Resultados}

Perfil de los pacientes. Prevalencia del dolor: Las características de los pacientes se reflejan en la tabla 1. Hallamos una elevada prevalencia del dolor fuera de la sesión de diálisis $(82.1 \%)$, así como un elevado porcentaje de pacientes (65\%) que recibía analgesia. Sin embargo, mediante la EVA realizada en un día de no diálisis, el porcentaje de pacientes que refería dolor era algo inferior (71.8\%). Los días con dolor a la semana fueron $4.75 \pm 2.5$, presentándose el dolor durante el día (43.7\%) o de día y de noche $(34.3 \%)$.

\begin{tabular}{l|l} 
Características & Pacientes \\
Sexo & $15 \mathrm{M} / 17 \mathrm{H}$ \\
\hline Edad media & $66.7 \pm 13.6$ años \\
\hline Estado civil: & $18(56.2 \%)$ \\
Casado & $6(18.7 \%)$ \\
Viudo & $5(15.6 \%)$ \\
Soltero & $3(9.3 \%)$ \\
\hline Separado & \\
\hline Enfermedad renal primaria: & $10(31.2 \%)$ \\
\hline Diabetes mellitus & $7(21.8 \%)$ \\
Poliquistosis renal & $7(21.8 \%)$ \\
No filiada & $5(15.6 \%)$ \\
Otras & $2(6.2 \%)$ \\
Glomerulonefritis & $1(3.1 \%)$ \\
\hline Nefropatía intersticial & \\
\hline Comorbilidad: & $23(71.8 \%)$ \\
Hipertensión arterial & $12(37.5 \%)$ \\
Cardiopatía isquémica & $6(18.7 \%)$ \\
Neoplasia & $3(9.3 \%)$ \\
Enf. Arterial periférica & $2(6.2 \%)$ \\
\hline Accidente cerebrovascular & \\
\hline Tiempo en hemodiálisis: & $61.5 \pm 60.3$ meses \\
Pacientes con dolor: & $47.8 \pm 43.8$ meses \\
Pacientes sin dolor: & $(p=N S)$ \\
\hline Pacientes con dolor fuera & $26(81.2 \%)$ \\
\hline de diálisis & $21(65.6 \%)$ \\
\hline Medicación analgésica & \\
\hline &
\end{tabular}

Tabla 1. Características de los pacientes.

Causas y localización del dolor: El tipo más frecuente fue el dolor músculo-esquelético (21 pacientes), aunque destaca la alta prevalencia del dolor relacionado con el procedimiento (12), siendo el do- lor isquémico (8) la tercera causa y a localización más prevalente las extremidades inferiores $(62,5 \%)$ y la región dorsal.

Severidad y manejo del dolor: Las puntuaciones de las diferentes escalas se pueden observar en las tablas 2 (EVA, McGill y PMI) y 3 (BPI). La gran mayoría de pacientes presentaba un dolor leve 0 moderado, y en ningún caso se registró un dolor severo (EVA superior a 7.5). Según el BPI, la mitad de los pacientes refería tener dolor en el momento de la entrevista, evaluado como de grado leve a moderado. De entre las actividades de la vida diaria, la menos interferida por el dolor fue la relación social con la gente $(p<0.05)$, en comparación con la actividad general y el desplazamiento/caminar. En cuanto a la medicación (tabla 4), la totalidad de pacientes que recibían analgesia estaban tratados con paracetamol, algunos de ellos además recibían opiáceos menores (tramadol o codeína), y en un pequeño porcentaje, opiáceos mayores (buprenorfina). El uso de medicación coadyuvante (sertralina o gabapentina) se registró en un tercio de los pacientes. La aplicación de medidas físicas como el calor, los cambios posturales o los masajes mejoraban la sintomatología en el $56 \%$, $43.7 \%$ y $31 \%$ de casos respectivamente. No obstante, el porcentaje de pacientes "sufridores" fue del $84.3 \%$.

\begin{tabular}{l|l|l}
\multicolumn{1}{c|}{} & Valor medio & $2.41 \pm 2.13$ \\
\hline \multirow{4}{*}{ EVA } & Valores $0-2.5$ & $53.1 \%$ \\
\hline & Valores $2.6-5$ & $37.5 \%$ \\
\hline & Valores $5.1-7.5$ & $9.3 \%$ \\
\hline Valores $7.6-10$ & $0 \%$ \\
\hline \multirow{3}{*}{ MPQ } & PRI-total & $22.84 \pm 11.81$ \\
\hline \multirow{4}{*}{ PMI } & PPI & $1.61 \pm 0.76$ \\
\hline & NPE & $10.28 \pm 4.23$ \\
\hline & Valor medio & $0.34 \pm 1.03$ \\
\hline & Valores positivos & $34.3 \%$ \\
\hline & Valores neutros & $46.8 \%$ \\
\hline & Valores negativos & $18.75 \%$ \\
\hline & &
\end{tabular}

Tabla 2. Puntuaciones de las diferentes escalas: EVA, McGill (MPQ) y PMI.

Relaciones entre distintas variables: No encontramos correlación entre las características clínicas de los pacientes y las diferentes escalas del dolor, ex- 
cepto para el tiempo de permanencia en diálisis, que se correlacionaba con el nivel de la EVA $(r=0.36$, $p<0.05)$, el PRI-total ( $r=0.38, p<0.05$, figura 1 ) y el número de palabras escogidas del $\mathrm{MPQ}(r=0.39$, $\mathrm{p}<0.05$ ). Asimismo, los niveles plasmáticos de PTHi se relacionaron con la puntuación de la EVA $(r=0.44, p<0.05$, figura 2), el PRI-total $(r=0.32$, $p=0.05$ ), y la interferencia con el desplazamiento/caminar del BPI $(r=0.35, p<0.05)$.

\begin{tabular}{|c|c|c|}
\hline $\begin{array}{l}\text { Dolor }(n=26) \\
\text { Dolor hoy }\end{array}$ & Si: $50 \% /$ No: $50 \%$ & Puntuaciones $(0-10)$ \\
\hline $\begin{array}{l}\text { El peor dolor } \\
\text { ultimas } 24 \mathrm{~h}\end{array}$ & & $4.46 \pm 3.7$ \\
\hline $\begin{array}{l}\text { El menor dolor } \\
\text { ultimas } 24 \mathrm{~h}\end{array}$ & & $1.56 \pm 2.09$ \\
\hline $\begin{array}{l}\text { El dolor medio } \\
\text { ultimas } 24 \mathrm{~h}\end{array}$ & & $3.28 \pm 2.72$ \\
\hline Dolor actual & & $2.09 \pm 2.7$ \\
\hline Medicación recibida & Si : $59.3 \% / \mathrm{No}: 40 \%$ & \\
\hline Grado de mejoría & & $6.72 \pm 3.1$ \\
\hline \multirow{7}{*}{$\begin{array}{l}\text { Interferencia } \\
\text { a ctividades } \\
\text { de la vida diaria }\end{array}$} & Actividad general & $4.43 \pm 3.45$ \\
\hline & Desplazamiento/caminar & $4.96 \pm 3.65$ \\
\hline & Tareas habituales & $3.62 \pm 3.09$ \\
\hline & Carácter & $3.8 \pm 3.31$ \\
\hline & Relación social & $1.77 \pm 2.55(p<0.01)^{*}$ \\
\hline & Ocio & $3.28 \pm 3.6$ \\
\hline & Descanso & $2.62 \pm 3.46$ \\
\hline
\end{tabular}

* Respecto a actividad general y desplazamiento/caminar

Tabla 3. Severidad del dolor según las puntuaciones del BPI.

\begin{tabular}{l|c|c}
\multirow{2}{*}{ Fármacos } & Ninguno & $34.3 \%$ \\
\cline { 2 - 3 } & No opiáceos (paracetamol) & $65.6 \%$ \\
\hline & Opiáceos menores & $25 \%$ \\
\hline Vías de administración & Opiáceos mayores & $15.6 \%$ \\
\hline & Coadyuvantes & $31.2 \%$ \\
\hline Tipo de administración & Oral & $65.6 \%$ \\
\hline Cumplimiento terapéutico & Pauta fija: $27.2 \%$ & \\
\hline & A demanda: $72.7 \%$ & \\
\hline & $70 \%$ & \\
\hline
\end{tabular}

Tabla 4. Medicación recibida.

\section{Relación tiempo de diálisis y puntuación del PRI-total (MPQ)}

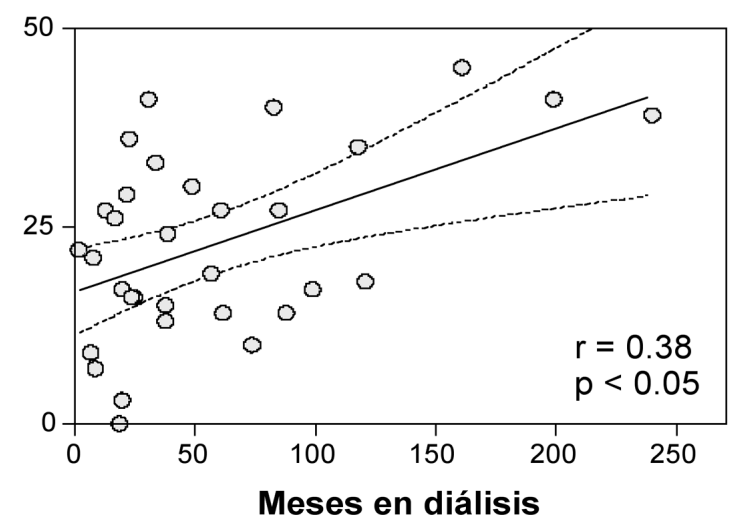

Figura 1.

\section{Relación entre el valor de la EVA y los niveles de PTHi}

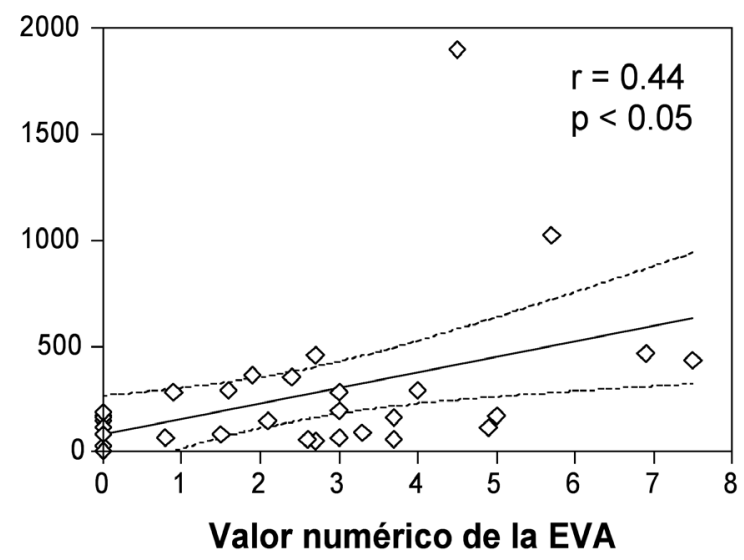

Figura 2.

\section{Discusión}

Nuestro estudio muestra una alta prevalencia del dolor en la población de HD. EI 82.1\% de los pacientes afirmaron tener dolor en casa y el $50 \%$ expresaron haber tenido una experiencia dolorosa en las últimas 24 horas. Únicamente 7 pacientes negaron tener dolor en casa y en 9 casos (28.12\%) la EVA fue 0. Estos datos confirman la línea de estudios anteriores que nos muestran una imagen del dolor como un síntoma común y generalizado en las salas de HD con el que pacientes y profesionales convivimos día a día ${ }^{2,3}$. 
En cuanto a su intensidad, la mayoría de los pacientes lo califican de leve a moderado, existiendo un porcentaje bajo de dolor severo. En otros estudios la intensidad del dolor descrita fue mayor ${ }^{2,3}$, si bien hemos encontrado en nuestra población un uso de fármacos superior al de los estudios mencionados.

En relación a la causalidad, se halló un alto porcentaje de dolor músculo-esquelético, muy similar al descrito en otras series de pacientes ${ }^{2} 0$ incluso en la población general ${ }^{6}$. Sin embargo, en un estudio anterior $^{3}$ se describe una menor proporción de pacientes con dicho tipo de dolor, posiblemente debido a la distinta metodología utilizada. A este respecto, el ambiente de recogida de información podría ser un factor influyente en la respuesta del paciente, lo que justificaría futuras investigaciones destinadas a homogeneizar la metodología de valoración del dolor en los pacientes en HD. Independientemente del momento y lugar escogidos para recabar la información, parece haber un factor común en todos estos estudios: los pacientes identifican en el propio procedimiento de la hemodiálisis una causa o foco de dolor, que además supone una de las principales causas de dolor en estos pacientes.

Los valores del PMI orientan hacia una utilización razonable de la medicación analgésica en la mayoría de pacientes. Este dato está en consonancia con el elevado cumplimiento terapéutico descrito. Por otro lado, no es de extrañar que, siendo la principal causa de dolor el de origen músculo-esquelético, las medidas físicas como los cambios posturales o la aplicación de calor local hayan mostrado una eficacia similar a la de los fármacos. Sería conveniente realizar más estudios para valorar el papel específico que otras medidas no farmacológicas tendrían en el tratamiento del dolor.

Se encontró una relación estadísticamente significativa entre el tiempo de permanencia en HD y la intensidad del dolor, así como con sus características cualitativas, independientemente de la edad y el sexo de los pacientes. Aunque no todos los estudios consultados han podido encontrar dichas relaciones, existen indicios en la literatura que apuntan en este sentido ${ }^{1}$. Sería lógico pensar que un mayor tiempo de permanencia en hemodiálisis pudiera favorecer la aparición de ciertas patologías como la osteodistro- fia renal o la amiloidosis de diálisis que podrían contribuir a la presencia de dolor.

Los niveles de PTHi se relacionaron con los valores de la EVA y las puntuaciones del MPQ, así como con una mayor interferencia del dolor en la variable desplazamiento/caminar. No hemos encontrado otras referencias en la literatura que hayan descrito estas relaciones. Nos parece razonable pensar que un mayor grado de hiperparatiroidismo se acompañe de una mayor intensidad y mayor valoración cualitativa del dolor, y dificulte a los pacientes su deambulación. Convendría, en futuras investigaciones, confirmar estos hallazgos y profundizar en el impacto que pudiera tener el hiperparatiroidismo en la elevada prevalencia de dolor músculo-esquelético en las poblaciones de HD.

Hay que mencionar algunas limitaciones en este estudio. Por un lado, hemos de entender siempre el dolor como un síntoma multidimensional en el que la dimensión psicológica juega un papel relevante. La relación entre depresión y dolor ha sido ampliamente documentada en otros grupos de pacientes ${ }^{7,8}$, pero hemos de considerar que su análisis queda, a nivel metodológico, fuera de nuestro alcance. Son necesarios otros estudios que aporten luz a este tema en el campo de la HD. Por otro lado, hemos de recalcar que los instrumentos de valoración utilizados en el presente estudio no han sido diseñados específicamente para las necesidades valorativas del paciente en HD, por lo que sería necesaria su validación en poblaciones más amplias de este tipo de pacientes.

\section{Conclusiones}

- El dolor es un problema común en los pacientes de HD. En nuestra experiencia, este síntoma se presenta con mucha frecuencia $(82.1 \%)$, es de intensidad leve a moderada, cotidiano, preferentemente diurno y de duración prolongada. Es un factor que limita ciertas actividades de la vida diaria, principalmente el desplazamiento/caminar y la actividad general.

- El dolor de origen músculo-esquelético es la causa más frecuente en nuestra población y afecta predominantemente a las extremidades inferiores. 
- Hay indicios que parecen apuntar hacia un uso razonable de las terapias farmacológicas en el tratamiento del dolor. A pesar de esto, queda todavía un largo camino por recorrer para conseguir un control óptimo del dolor en estos pacientes.

\section{Bibliografía}

1. Marín MT y otros. El dolor crónico, un estudio en pacientes en hemodiálisis. En: Libro de comunicaciones presentadas al XXIII Congreso de la SEDEN. 1998.

2. Davison SN. Pain in hemodiálisis pacients: prevalence, cause, severity and management. American Journal of Kidney Diseases. 2003; 6(42):1239-1247.

3. Rodríguez MA y otros. Evaluación y manejo del dolor intradiálisis. Rev Soc Esp Enferm Nefrol. 2006; 9(2):65-70.
4. Daut RL, Cleeland ES, Flanery RC. Development of the Wisconsin Brief Pain questionnaire to asses pain in cancer and other desease. Pain. 1983; 17:197-210.

5. Melzack R. The McGill pain Questionnaire: major propertiesand scoring methods. Pain. 1975; 1:277-282.

6. Lázaro C y otros. The development of Spanish Questionnaire for assesing pain: preliminary data concerning reability and validity. EU J Psychol Assessment. 1994; 10(2):145-151.

7. Catalá I y otros. Prevalencia de dolor en la población española: encuesta telefónica en 5000 hogares. Eur J Pain. 2002; 6:133-140.

8. Sorkin BA y otros. Chronic pain in old and young patients: differences appear less important than similarities. J Gerontol. 1990; 45:64-68. 